\title{
STUDIO DEL TERREMOTO DELLE ISOLE LIPARI DEL 13 APRILE 1938
}

\author{
P. Caloi - M. Giongi
}

\section{Parte Prima}

Premessa. - La maggior parte dei terremoti cosidetti profondi o plutonici, aventi cioè origine al di sotto della discontinuità di Mohorovic, hanno i loro epicentri disseminati lungo la zona circum-pacifica $\left(^{1,2.3}\right)$; precisando si può affermare che la totalità dei terremoti profondissimi (con profondità che vanno dai 300 ai $700 \mathrm{~km}$ ) ed il $90 \%$ dei terremoti con profondità dai 60 ai $300 \mathrm{~km}$ ca, hanno i loro epicentri in detta fascia; il restante $10 \%$ ca dei terremoti a profondità intermedia sono distribuiti lungo un arco che comprende il Mediterraneo orientale e la zona trans-asiatica.

Attraverso gli studi di Critikos $\left({ }^{4}\right)$ e Gutenberg $\left({ }^{3}\right)$ è stata ormai accertata l'esistenza di ipocentri profondi (fino a $300 \mathrm{~km} \mathrm{ca}$ ) nel mare Egeo: ma ipocentri così profondi nel Mediterraneo centrale erano insospettati fino a quando precise determinazioni portarono ad attribuire il valore di $300 \mathrm{~km}$ alla profondità del terremoto delle Isole Lipari, avvenuto alle ore $02^{11} 45^{\mathrm{m}}$ del 13 aprile 1938.

Uno studio macrosismico sui dati di osservazione italiani relativi a detto terremoto fu condotto da G. Agamennone nel 1938 ( 5 ); egli contestò i valori delle coordinate ipocentrali comunicati dai bollettini di Strasburgo e della J.S.A., nonché quelli calcolati con i dati di alcune stazioni europee da Demetrescu $\left(^{0}\right)$, perché in apparente disaccordo con i risultati macrosismici. L'esame attento delle risultanze macrosismiche danno, invece, come si vedrà più sotto, una chiara conferma della notevole profondità ipocentrale di questo terremoto che all'eminente sismologo dovette apparire eccezionale, per il luogo ove il sisma elble a verificarsi.

E chiaro quindi l'interesse che presenta questo nostro lavoro, non solo per tutte le evidenze che possono trarsi dallo studio dei terremoti profondi in genere, nei riguardi cioè della natura e del meccanismo della loro origine, nonché della costituzione, stato e profondità degli strati più interni del pianeta, ma anche per $i$ risultati che po- 
tranno dedursi da un'indagine particolareggiata di questo terremoto in connessione col fatto che esso si è verificato al centro del Mediterraneo, in una zona tanto complessa nei riguardi della geologia superficiale e profonda. L'epicentro risulta situato infatti lungo l'allineamento della fascia vulcanica attiva che dal Vesuvio attraverso lo Stromboli va fino all'Etna.

E bene precisare intanto che il terremoto in questione non $i$ avvenuto in concomitanza con un'aceresciuta attivita di alcuno di detti vulcani e che in genere per terremoti tanto profondi i da escludere una diretta azione di causa ed effetto tra $\mathrm{i}$ due fenomeni.

E noto infatti (3) che dallo studio della sismicità della terra, attraverso l'indagine sulla natura dei terremoti (vuleanici, tettonici, plutonici) e sulle loro caratteristiche fondamentali (profondità, energia, ecc.) in rapporto alla loro distribuzione nel tempo e nello spazio, nonché alle loro relazioni con eventuali anomalie geofisiche (in particolare gravimetriche) e la struttura più o meno profonda delle varie regioni del pianeta, risulta che i terremoti che si verificano nelle zone di vulcani attivi si possono distinguere in tre categorie:

a) terremoti superficiali aventi un carattere più o meno strettamente locale e direttamente collegati ad una fase esplosiva, o cruttiva, o preeruttiva, in atto, dei vulcani; essi a causa della loro limilala energia si propagano a distanze relativamente hrevi, ed in genere si sussegruono in serie uno dopo l'altro per un certo periodo di tempo, a intervalli più o meno brevi;

b) terremoti di origine tettonica, indipendenti dall'atlività del vulcano;

c) terremoti con profondita fino a $200 \mathrm{~km}$ circa in concomitanza o meno di un'attività eruttiva dei vulcani; (nel primo caso, in ogni modo, non in relazione di causa ed effettol i da pensare che probabilmente entrambi i fenomeni sono effetto di un'unica causa ancora non identificata.

Il terremoto oggetto di questo studio avrenuto, come si i detto, alla eccezionale profondita di $300 \mathrm{~km}$ in una regione come quella in questione, costituisce quindi un easo notevole e l'interesse aumenta se si considera che la regione stessa i sede di una cospicua anomalia gravimetrica positiva, secondo Bouguer, pari a +240 mgal rispetto al valore normale calcolato con la formula internazionale. I calcoli per la riduzione isostatica di Hayford-Bowie (per $h_{c}=96 \mathrm{~km}$ ) delle misure gravimetriche danno per la zona in prossimita dellepicentro una anomalia di 100 mogal ca $\left({ }^{7}\right)$. 
La forte eccedenza positiva denuncia la mancanza di equilibrio isostatico della regione.

Le sopradette ragioni ci banno indotto a procedere ad uno studio

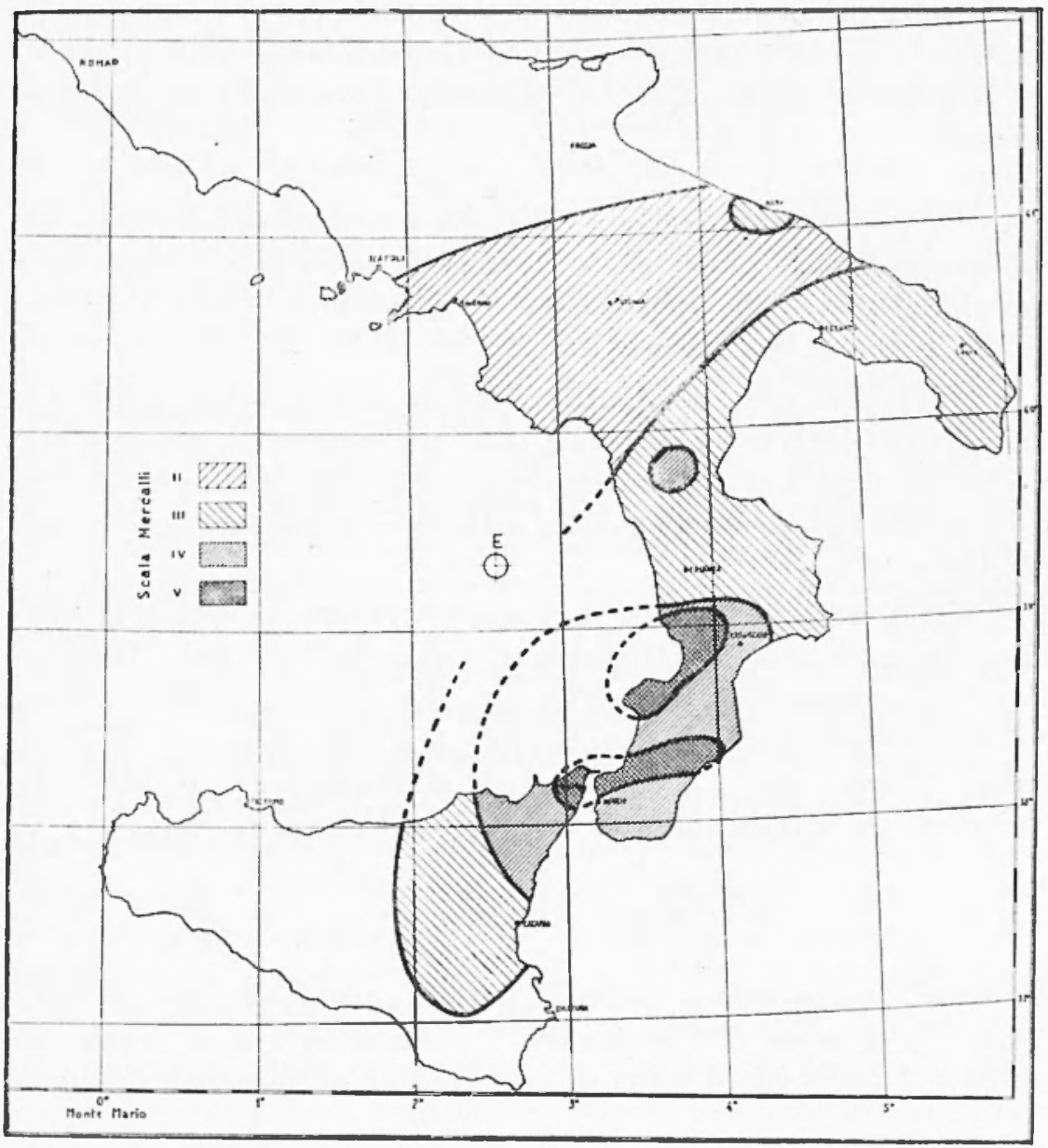

Fig. 1

accurato di questo terremoto. Come condizione indispensabile per l'ulteriore sviluppo dello studio, abbiamo creluto innanzi tutto necessario eseguire una revisione delle coordinate spazio-temporali.

Dette coordinate vennero già da altri $\left(^{6}\right)$ calcolate, ma con approssimazione insufficiente per i nostri scopi; abbiamo potuto invece ottenere la necessaria precisione dei risultati effettuando le misure diret- 
tamente su diverse decine di sismogrammi originali o copie fotografiche in wrandezza naturale che ci sono state cortesemente inviate da diversi Osservatorî del mondo a cui intendiamo esprimere il nostro doveroso ringraziamento.

Questa prima parte dello studio comprende quindi i procedimenti usati ed i risultati conseguiti per la determinazione delle coordinate geografiche dell'epicentro, la profonditì ipocentrale ed il tempo orinine.

1. Risultati macrosismici. - I risultati macrosismici vengono riepilogati nell'annessa cartina. Per notizie più dettagrliate a tal riguardo inviamo il lettore alle pubblicazioni di Agamennone (5). Ci limitiamo a notare solo quanto segue:

a) la irregolare distribuzione dell'intensiti e la sua eccentricita rispetto allepicentro in superficie sta a testimoniare il carattere profondo del terremoto; la distribuzione dell'energia sismica in superficie è infatti fortemente influenzata dalle discontinuitì geologiche degli strati più esterni;

b) l'entita della scossa in superficie, anche nei punti di marrgriore intensità, non ha oltrepassato il grado $V$ della scala Mercalli; ora, la "magnitudo " ( $\left(^{(}\right)$di detto sisma ha il valore di $M=63 / 1$ e a tal valore corrisponde un'energia, irragriata all'origine sotto forma di onde elastiche, pari a $E=10^{2: 3}$ erw, se si prende per $E$ il valore che si ottiene dalla relazione seguente data da Gutenberg (3):

$$
\log E=11,3+1,8 \mathrm{M} \text {. }
$$

la entiti, relativamente modesta, della intensità macrosismica nella regicne pleistosista confrontata con la effettiva energia allorigine del terremoto prova che si tratta di un terremoto ad ipocentro profondo.

2. Coordinate epicentrali. - I metodi analitici e mrafici per la determinazione delle coordinate epicentrali di terremoti lontani, basati sulla conoscenza dei tempi di arrivo delle onde di dilatazione consentono una approssimazione più o meno spinta che dipende da molteplici cause; se si fa astrazione dayli errori di osservazione e dalle inevitahili inesattezze nella determinazione dei tempi di inizio delle fasi, dovuta alla difficolta di discemere il preciso inizio, alla mancata uniformitì di scorrimento delle zone, arli errori degli oro- 
logi marca-tempo, ecc., la maggior parte dei metodi anzidetti richiede la conoscenza della profondità ipocentrale e della relativa dromocroma ed è proprio dalla scelta di quest'ultima che dipende l'approssimazione dei risultati. Per ottenere risultati più precisi si applica il metodo delle approssimazioni successive. È chiaro che, quand'anche fossero eliminate tutte le cause di errori sistematici, ci sarà sempre una serie di dati di osservazione non in perfetto accordo tra di loro che individueranno un insieme di punti disseminati in una piccola zona; col metodo dei minimi quadrati, si potrà determinare poi l'epicentro più probabile.

Esiste peraltro un metodo dovuto a Galitzin (9) il quale è del tutto indipendente dalle dromocrone e dalla profondità ipocentrale; esso si può usare, quando naturalmente sussistono le condizioni necessarie per la sua applicazione, specialmente quando non si conosce la pro. fondità del fuoco o pur conoscendola non ci si vuol servire di dromocrone empiriche prestabilite, allo scopo di mettersi nelle condizioni della magriore indipendenza possibile da posizioni che comunque possono influenzare $\mathrm{i}$ risultati oltre lo strettamente necessario. Esso da altri ricercatori $\left({ }^{10,11,12}\right)$ è stato usato con successo nello studio di terremoti profondi.

Abbiamo creduto opportuno di dar molto credito a questo metodo per lo studio del nostro terremoto specialmente perché abbiamo voluto ignorare completamente i valori approssimati già noti della sua profondità ipocentrale; $\dot{e}$ vero che il metodo in questione suppone che la propapazione sia isotropa e non tiene quindi conto delle inomogeneità degli strati della crosta terrestre, ma questa ipotesi non è certamente meno restrittiva di altre ipotesi su cui si basano altri metodi, ben più numerose e non meno arbitrarie. Si può affermare quindi che, quando esso è applicabile, l'approssimazione dei risultati non è minore di quella conseguibile con altri metodi. Del resto, alla pari con gli altri metodi, mediante un numero sufficiente di combinazioni si può supporre che avvenga una compensazione statistica degli errori che derivano dall'ipotesi della propagazione isotropa.

Per fini pratici diamo una breve esposizione della applicazione analitica del metodo adottato.

Avendo a disposizione i tempi di tragitto della fase $P$ in una serie di Osservatorî, si scelgono coppie di stazioni in cui $\mathrm{i}$ cerchi massimi passanti per esse e per l'epicentro siano divaricati il più possibile ed in cui i tempi di arrivo della $P$ siano uguali. 


\section{TABELLA I}

Valori di $\delta \backslash\left(\right.$ in $\mathrm{km}$ ) corrispondenti a $\delta \mathrm{t}=10^{\mathrm{ka}}$ alle varie distanze A (in gradi) per le diverse profondità h (in unità $\mathrm{R}$ ) dedotti dalle dromocrome per le $\mathrm{P}$ di Jeffreys "Bullen.

\begin{tabular}{|c|c|c|c|c|c|c|c|c|c|c|c|c|}
\hline & sup. & 0,00 & 0,01 & 0,02 & 0,03 & 0,04 & 0,05 & 0,06 & 0,07 & 0,08 & 0,09 & 0,10 \\
\hline $10^{\circ}$ & 81.0 & 81.3 & 83.0 & 84.9 & 87.0 & 89.7 & 92.6 & 95.8 & 10.4 & 109.0 & 113.4 & 120.8 \\
\hline $20^{\circ}$ & 107.0 & 107.0 & 109.0 & 111.0 & 112.0 & 113.0 & 115.0 & 116.0 & 118.2 & 120.8 & 122.1 & 123.5 \\
\hline $30^{\circ}$ & 126.3 & 124.8 & 126.3 & 126.3 & 127.7 & 127.7 & 129.2 & 129.2 & 129.2 & 130.7 & 130.7 & 132.3 \\
\hline $40^{\circ}$ & 135.5 & 133.9 & 135.5 & 135.5 & 135.5 & 135.5 & 137.2 & 137.2 & 137.2 & 138.9 & 138.9 & 140.6 \\
\hline $50^{\circ}$ & 146.2 & 146.2 & 146.2 & 146.2 & 148.2 & 149.9 & 149.9 & 149.9 & 149.9 & 152.2 & 152.2 & 154.3 \\
\hline $60^{3}$ & 163.4 & 163.4 & 163.4 & 163.4 & 165.8 & 165.8 & 168.3 & 168.3 & 168.3 & 168.3 & 170.9 & 170.9 \\
\hline $70^{\circ}$ & 182.1 & 182.1 & 185.2 & 185.2 & 185.2 & 185.2 & 185.2 & 188.3 & 188.3 & 188.3 & 191.6 & 191.6 \\
\hline $80^{\circ}$ & 209.6 & 209.6 & 209.6 & 209.6 & 209.6 & 213.7 & 213.7 & 213.7 & 213.7 & 213.7 & 217.9 & 217.9 \\
\hline $90^{\circ}$ & 241.5 & 236.4 & 236.4 & 236.4 & 241.5 & 241.5 & 241.5 & 241.5 & 241.5 & 241.5 & 241.5 & 241.5 \\
\hline $100^{\circ}$ & 246.9 & 246.9 & 246.9 & 246.9 & 246.9 & 246.9 & 252.5 & 252.5 & 252.5 & 252.5 & 252.5 & 252.5 \\
\hline
\end{tabular}

Nella ipotesi della propagazione isotropa, come dianzi si è detto, l'epicentro si troverà sul cerchio massimo che taglia ortogonalmente l'arco di cerchio massimo congiungente ogni coppia di stazioni; le intersezioni di questi cerchi in genere, per ovvie ragioni, non coincideranno, ma costituiranno una serie di punti sparsi in una zona di limitata estensione, da cui potrà dedursi l'epicentro più probabile.

La determinazione dell'epicentro si può eseguire anche nel caso che i tempi di tragitto delle $P$ nelle coppie di stazioni non siano uguali ma la loro differenza non superi una diecina di secondi al massimo. Infatti, se la differenza dei tempi si mantiene entro questi limiti la corrispondente differenza di distanza $(\delta /)$ delle stazioni dall'epicentro non è molto influenzata dalla profondità, specialmente se $\Lambda$ è grande.

Per comodità di calcolo nella tabella $I$ vengono riportati $i$ valori di $\delta \Lambda$ (incremento della distanza epicentrale in $\mathrm{km}$ ) corrispondenti ad una differenza nei tempi di tragitto delle onde $P(\partial t)$ uguale a 10 sec per le varie profondità ipocentrali, da $h=0$ fino al valore di 660 km ca; detti valori sono stati tratti dalle tabelle di Jeffreys e Bullen $\left({ }^{13}\right)$; (essi possono interpolarsi linearmente senza errori sensibili).

Pertanto nel caso in cui tra i tempi di arrivo delle $P$ di una coppia di stazioni (aventi rispetto all'epicentro azimut molto divaricati) esista una piccola differenza $(\delta t<10 \mathrm{sec})$ si potrà sostituire alle coordinate di una delle stazioni quelle di una stazione fittizia situata pì̀ avanti o più indietro rispetto alla stazione reale di un tratto pari a $\delta 1$, corrispondente a $\delta t$; il tratio $\delta \Delta$ va preso sullo stesso arco di cerchio 
massimo passante per la stazione reale e l'epicentro. Il procedimento solito si applichera allora alla coppia formata da una delle stazioni reali e dalla stazione fittizia. Se si indica con "c l'azimut (rispetto ad un epicentro provvisorio) della stazione da sostituire con la stazione fittizia, dal triangolo sferico Polo Nord, Epicentro, Stazione, con notazioni evidenti, si ha:

$$
\operatorname{sen} \alpha_{c}=\frac{\operatorname{sen}\left(\lambda_{c}-\lambda_{B}\right) \cos \rho_{B}}{\operatorname{sen} \Delta}
$$

La latitudine $\left(\psi^{\prime}\right)$ e la longitudine $\left(\hat{h}^{\prime}\right)$ del nuovo punto (stazione fittizia) sullo stesso arco di cerchio massimo alla distanza: $\Lambda^{\prime}=\Delta+\delta \Delta$ sono date dalle relazioni che seguono:

$$
\begin{gathered}
\operatorname{sen} \varphi^{\prime}=\cos \Delta^{\prime} \operatorname{sen} \varphi_{\mathrm{e}}+\operatorname{sen} \Delta^{\prime} \cos \varphi_{\mathrm{c}} \cos \alpha_{\mathrm{c}} \\
\cos \left(\lambda_{\mathrm{c}}-\lambda^{\prime}\right)=\frac{\cos \Delta^{\prime}-\operatorname{sen} \varphi_{\mathrm{e}} \operatorname{sen} \varphi^{\prime}}{\cos \varphi_{\mathrm{c}} \cos \varphi^{\prime}}
\end{gathered}
$$

Ottenuta allora una serie di coppie di stazioni (reali o fittizie) soddisfacenti alla richiesta condizione di uguale distanza epicentrale, si passa alla determinazione delle nuove coordinate epicentrali col metodo dei minimi quadrati, secondo il procedimento sopra ricordato.

Se si proietta la superficie terrestre, dal centro della terra su un piano parallelo al piano equatoriale, tutti i cerchi massimi si trasformeranno in rette. In particolare, l'equatore è la retta all'infinito del piano stesso.

Se $\lambda_{s_{1}}, q_{s_{1}}$ e $i_{s_{2}}, f_{s_{2}}$ sono le coordinate della $s^{\text {ma }}$ coppia di stazioni wià corretta, si ha:

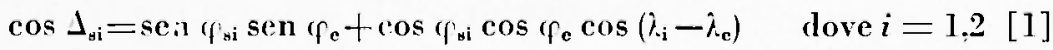

e poiche $\Delta_{q_{1}}=\Lambda_{s_{0}}=\Lambda_{*}$ uguagliando $i$ secondi termini delle $[1]$, si ottiene che il punto di coordinate $i_{n}$. ' fe sulla sfera si proietta sul piano nel punto di coordinate:

$$
\begin{aligned}
& x=\operatorname{cotg} \varphi_{e} \cos \gamma_{e} \\
& y=\operatorname{cotg} \gamma_{e} \operatorname{sen} \gamma_{c}
\end{aligned}
$$

ed il cerchio massimo equidistante dai due punti $S_{\mathrm{v}_{1}}, S_{\mathrm{sg}}$ ¿̀ rappresentato in proiezione la una retta:

$$
p_{s} x+q_{*} y=r_{*}
$$


dove le costanti $p_{.} . q_{\mathrm{v}}, r_{\mathrm{b}}$ corrispondono ai seguenti valori:

$$
\begin{aligned}
& p_{\mathrm{s}}=\cos \varphi_{\mathrm{s}_{1}} \cos \lambda_{\mathrm{o}_{1}}-\cos \varphi_{\mathrm{s} 2} \cos \lambda_{\mathrm{s} 2} \\
& q_{\mathrm{o}}=\cos \varphi_{\mathrm{s}_{1}} \operatorname{sen}{\lambda_{\mathrm{i}_{\mathrm{i}}}}_{1}-\cos \varphi_{\mathrm{B}, \mathrm{n}} \operatorname{sen} \lambda_{\mathrm{g}} \\
& r_{\mathrm{s}}=\operatorname{sen} \varphi_{\mathrm{B}_{2}}-\operatorname{sen} \varphi_{\mathrm{s}_{1}}
\end{aligned}
$$

Si avrà quindi il sistema di equazioni simultanee:

$$
p_{s} x+q_{s} y^{\prime}=r_{s} \quad \text { con } \quad s=1,2, \ldots n
$$

la cui soluzione mediante il metodo dei minimi quadrati darà per le incognite $i$ valori seguenti:

$$
\begin{aligned}
& x=A-a \\
& y=B
\end{aligned}
$$

da cui, mediante le [2], si ottengrono le coordinate cpicentrali:

$$
\begin{aligned}
& \varphi_{\mathrm{e}}=\operatorname{arccotg}\left(A^{-}+B^{2}\right)^{\frac{1}{2}} \\
& \lambda_{3}=\operatorname{arccotg} \frac{A}{B} .
\end{aligned}
$$

Se si vogrliono queste grandezze con grli errori medi, ricordando che l'errore medio di una funzione $f\left(z_{1}, z_{2}, \ldots z_{i}\right)$ di quantita osservate $z_{1}, z_{2}, \ldots z_{n}$, dove:

è dato da:

$$
z_{\mathrm{i}}=A_{\mathrm{i}}+a_{\mathrm{i}}
$$

$$
\eta=\sqrt{\sum_{1}^{\mathrm{n}}\left(\frac{\partial f}{\partial z_{\mathrm{i}}}\right)_{A_{\mathrm{i}}}^{2} a_{\mathrm{i}}^{2}}
$$

abbiamo in definitiva:

$$
\left\{\begin{array}{l}
\overline{\varphi_{\mathrm{c}}}=\operatorname{arccotg}\left(A^{2}+B^{2}\right)^{\frac{1}{2}} \pm \frac{1}{1+\left(A^{2}+B^{2}\right)} \sqrt{\frac{a^{2} A^{2}+b^{2} B^{2}}{A^{2}+B^{\circ}}} \\
\overline{\lambda_{\mathrm{c}}}=\operatorname{arctg} \frac{B}{A}+\frac{\sqrt{B^{2} a^{2}+A^{2} b^{\circ}}}{A^{2}+B^{2}}
\end{array}\right.
$$

Per applicare il metodo abbiamo scelto, tra tutto il materiale di osservazione che ci è pervenuto, i dati di 12 stazioni con $d$ compresa tra $10^{\circ}$ e $17^{\circ}$ ca combinate a coppie come viene sotto descritto. I dati riferentisi a questi osservatori vengono dati nella tabella II. 
TABella II

\begin{tabular}{|c|c|c|c|c|c|c|c|c|}
\hline N. & Osservatorio & $\lambda$ & $-\int_{0}^{T} \varphi$ & $\begin{array}{c}\Delta \\
\text { provv. }\end{array}$ & h & $\begin{array}{l}T_{f} \\
\mathbf{m}\end{array}$ & $s$ & $\frac{\alpha_{c}}{\text { dall'ep. provv. }}$ \\
\hline 1 & Istambul & $29^{\circ} 03^{\prime}, 5 \mathrm{E} \mathrm{Gr}$. & $41^{\circ} 03^{\prime}, 1 \mathrm{~N}$ & 1205 & (1) 2 & & 17.6 & $+75^{\circ} 45^{\prime}, 9$ \\
\hline 2 & Cheb & $\left.12^{\circ} 22^{\prime}, 6\right)$ & $\left.50^{\circ} 04^{\prime}, 8\right)$ & 1228 & 》 & ) & 21.2 & - $\quad 9011^{\prime}, 5$ \\
\hline 3 & Jen:ı & $11^{\circ} 35^{\prime}, 0 》$ & $\left.50^{\circ} 56^{\prime}, 1\right)$ & 1332 & ” & ” & 33.0 & $-10^{\circ} 47^{\prime}, 2$ \\
\hline 4 & Alicante & ()$^{\circ} 29^{\prime}, 2 \mathrm{WGr}$. & $38^{\circ} 21^{\prime}, 3 »$ & 1356 & $\triangleright$ & » & 36.0 & $-88^{\circ} 46^{\prime}, 3$ \\
\hline 5 & Gollinga & $9 " 57^{\prime}, 9 \mathrm{E} \mathrm{Gr}$. & $51^{\circ} 32^{\prime}, 8$ ) & 1431 & $"$ & & 42.6 & $-14^{\circ} 30^{\prime}, 9$ \\
\hline 6 & Yalt: & $34^{\circ} 09^{\prime}, 3$ ) & $\left.4.4^{\circ} 29^{\prime}, 2 \quad\right)$ & 1683 & » & 49 & 07.0 & $+63^{\circ} 26^{\prime}, 0$ \\
\hline 7 & Amburgo & 90 & $53^{\circ} 33^{\prime}, 6$ ”) & 1643 & » & ” & 08.5 & $-12^{\circ} 02^{\prime}, 2$ \\
\hline 8 & Sinferopoli & $34^{\circ}\left(07^{\prime}, 0\right)$ & $44^{\circ} 57^{\prime}, 0 \mathrm{~m}$ & 1693 & $»$ & ") & 12.8 & $+61^{\circ} 40^{\prime}, 9$ \\
\hline 9 & Helwa & $\left.31^{\circ} 20^{\prime}, 5\right)$ & $\left.29^{\circ} 51^{\prime}, 6\right)$ & 1810 & $\eta$ & $»$ & 22.0 & $+119^{\circ} 56^{\prime}, 5$ \\
\hline 10 & Kew & (0) $18^{\prime}, 8$ WGr. & $51^{\circ} 28^{\prime}, 1 \quad »$ & 1814 & $»$ & $»$ & 22.0 & $-36^{\circ} 16^{\prime}, 5$ \\
\hline 11 & Copenaghen & $12^{\circ} 27^{\prime}, 0 \mathrm{E} \mathrm{Gr}$. & $55^{\circ}+1 l^{\prime},(0)$ & $18 \cdot 43$ & ” & $"$ & 24.8 & - $5^{\circ} 15^{\prime}, 8$ \\
\hline 12 & lind & $13^{\prime \prime \prime} 11^{\prime}, 2$ ) & $55^{\circ}+11^{\prime}, 9 》$ & 1839 & » & $"$ & 25.4 & $-\quad 3^{\circ} 48^{\prime}, 2$ \\
\hline
\end{tabular}

Per il calcolo della distanza provvisoria, che occorre per determinare le coordinate delle stazioni fittizie da sostituire ad alcune stazioni reali, siamo partiti da un ipotetico epicentro di coordinate: $\varphi_{\mathrm{e}}=39^{\prime \prime}, 2 \mathrm{~N}, /_{\mathbf{c}} 15^{\circ}=15^{\prime \prime}, 1 \mathrm{E}$ Gr. che è il baricentro di quattro punti già noti e riportati nella tabella III.

TABELLA III

\begin{tabular}{|c|c|c|c|c|c|c|}
\hline Autorita & $\varphi$ & $\lambda$ & $l_{1}$ & $\begin{array}{c}H_{0} \\
\mathbf{m}\end{array}$ & $s$ & $\begin{array}{c}h \\
\mathrm{~km}\end{array}$ \\
\hline Strasburgo & $39^{\circ}, 5 \mathrm{~N}$ & $15^{\circ}, 0 \mathrm{E} \mathrm{Gr}$. & 2 & 45 & 45 & - \\
\hline J.S.A. & $39^{\circ}, 4 \quad$ ") & $15^{\prime \prime}, 0 "$ & 2 & 45 & 54 & 300 \\
\hline U.S.C.G.S. & $38^{\circ}, 8 \quad$ & $15^{\circ}, 2 "$ & & - & & - \\
\hline Demetrescu & $\left.39^{\circ}, 1 \quad\right)$ & $\left.15^{\circ}, 3 \quad\right)$ & 2 & .45 & 53 & 226 \\
\hline
\end{tabular}

Le distanze sono state sempre calcolate in coordinate geocentriche e con i noti procedimenti della sismologia $\left({ }^{13}\right)$.

Le stazioni di cui alla tabella 111 sono state accoppiate a due a due sostituendo in ogni coppia ad una delle stazioni reali la corrispondente fittizia, ad uguale distanza (di tempo); il modo in cui è stato effettuato detto accoppiamento is riportato nella tabella IV. I nomi con asterisco rappresentano le stazioni sostituite.

E da osservare che gli errori rhe possono commettersi nello stabilire le coordinate delle stazioni fittizie, arretrando o avanzando 


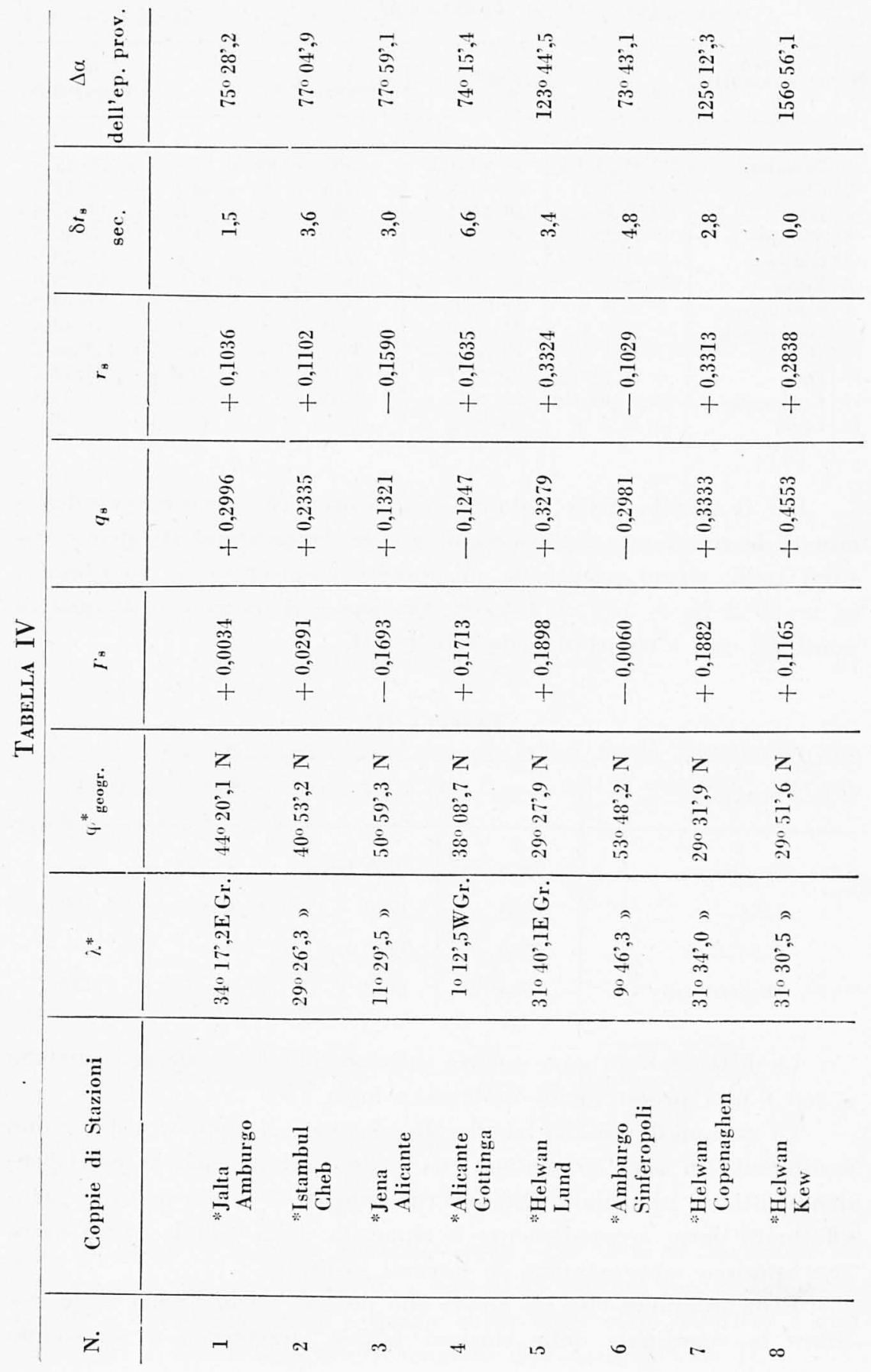


quelle reali, quando le differenze di tempo tra le coppie si mantengono inferiori ai 10 secondi e quando per il calcolo delle distanze di arretramento o avanzamento si adotta una dromocrona anche grossolanamente approssimata, sono dello stesso ordine di grandezza di quelli relativi alla lettura dei tempi sui sismogrammi. Infatti nel nostro caso le differenze di tempo $\delta t$ tra le coppie scelte sono sempre molto piccole; come si vede nella taljella IV, il massimo valore si ha per la coppia Alicante-Gottinga: $o r=6^{\circ}$. 6; avendo adottato la dromocroma corrispondente ad $h=0,05 R$ (dove $R=6338 \mathrm{~km}$ ) delle tavole di Jeffreys e Bullen (-) anche se la profondità fosse errata di $0,01 R$ l'errore corrispondereble a quello di 0,4 sec. nella lettura del sismogramma; in tutti gli altri casi, eguale errore nella valutazione della profonditì corrisponde a errori di lettura dei tempi sui sismogrammi molto inferiori a questo massimo e precisamente dell'ordine di 0.1 secondi.

Stabilite le equazioni normali del sistema [5]:

$$
\begin{aligned}
& {[p p] x+[p q] y=[p r]} \\
& {[p q] x+[q q] y=[q r]}
\end{aligned}
$$

sono stati ottenuti $\mathrm{i}$ seguenti valori per le incognite:

$$
\begin{array}{rlrl}
x & =1,18880 & y & =0,32315 \\
\mu_{x}= \pm \sqrt{\frac{\left[r r_{2}\right]}{(n-1)\left[p p_{1}\right]}}= \pm 0,00706 & \mu_{y}= \pm \sqrt{\frac{\left[r r_{2}\right]}{(n-1)\left[q q_{1}\right]}}= \pm 0,00322
\end{array}
$$

da cui mediante le [8] si deducono i seguenti valori per le coordinate dell'epicentro:

$$
\begin{gathered}
i=15^{\circ} \quad 12^{\prime}, 4=10^{\prime}, 3 \\
q_{\text {geoc. }}^{\prime}=39^{\circ} 06^{\prime}, 0=9^{\prime}, 3 \quad \text { T }_{\text {geografica }}=39^{\circ} 17^{\prime}, 3 \pm 9^{\prime}, 3
\end{gathered}
$$

Con i dati delle stesse stazioni di cui alla tabella II si è cercata la soluzione dello stesso problema con un metodo statistico di P. Caloi $\left.{ }^{15}\right)$; detto metodo consente di ottenere contemporaneamente, oltre alle coordinate $\lambda, \propto$ dell'epicentro, anche il tempo origine $H$ e la profondità ipocentrale $h$.

Detti $H^{\prime}, \lambda^{\prime}, \varphi^{\prime}, h^{\prime}$ certi valori approssimati comunque ottenuti delle grandezze che si cercano, chiamando con $t_{0}$ il tempo di propagazione di una certa fase (in genere la $P$ ) dall'ipocentro alla $s^{\mathrm{mm}}$ stazione re- 
gistratrice e con $T$. il tempo di arrivo della stessa fase effettivamente osservato, si ha:

$$
T_{\mathrm{o}}=t_{\mathrm{t}}+H
$$

Chiamando con $H, \lambda, q, h$ i valori veri cercati, si avrà

$$
H=H^{\prime}+\delta H^{\prime} \quad \lambda=\lambda^{\prime}+\delta \lambda^{\prime} \quad \uparrow=\varphi^{\prime}+\delta \varphi^{\prime} \quad h=h^{\prime}+\delta h^{\prime}
$$

poiché: $t_{s}=f(\lambda, \uparrow, h)$ sarà anche:

$$
t_{s}=f\left(\lambda^{\prime}, \varphi^{\prime}, h^{\prime}\right)+\frac{\partial f}{\partial \lambda^{\prime}} \delta \lambda^{\prime}+\frac{\partial f}{\partial \varphi^{\prime}} \delta \varphi^{\prime}+\frac{\partial f}{\partial h^{\prime}} \delta h^{\prime}
$$

E facile verificare che:

$$
\begin{aligned}
& \frac{\partial f}{\partial \lambda^{\prime}}=\frac{\partial f}{\partial \Lambda_{s}} \cdot \frac{\partial \Lambda_{\mathrm{s}}}{\partial \lambda^{\prime}}=\cdots \cos \varphi^{\prime} \operatorname{sen} \alpha_{\mathrm{a}} \cdot \frac{\partial f}{\partial J_{\mathrm{b}}} \\
& \frac{\partial f}{\partial \varphi^{\prime}}=-\frac{\partial j}{\partial \Lambda_{\mathrm{s}}} \frac{\partial \Lambda_{\mathrm{s}}}{\partial \varphi^{\prime}}=-\cos \alpha_{\mathrm{s}} \frac{\partial f}{\partial \Lambda_{.}}
\end{aligned}
$$

ove con $a_{y}$ si $\dot{e}$ indicato l'azimut della stazione $s^{\text {mit }}$ rispetto all'epicentro. Quindi dalle [9] tenendo presenti le [10] e [11] se si hanno $n$ stazioni si arranno le seguenti $n$ equazioni simultanec:

$$
\delta H^{\prime}-\cos \varphi^{\prime} \operatorname{sen} \omega_{\mathrm{a}} \frac{\partial f}{\partial S_{\mathrm{a}}} \delta \lambda^{\prime}-\cos \left(\boldsymbol{t}_{\mathrm{a}} \frac{\partial f}{\partial \Lambda_{\mathrm{a}}} \delta \varphi^{\prime}+\frac{\partial f}{\partial h^{\prime}} \delta h^{\prime}=T_{\mathrm{a}}-H^{\prime}-f\left(\lambda^{\prime}, \varphi^{\prime}, h^{\prime}\right)\right.
$$

$\operatorname{con} s=1,2, \ldots n$.

Per la applicazione di detto metodo ablyiamo scelto $\mathrm{i}$ seguenti valori provisori per le coordinate spazio-temporali:

$$
\begin{array}{cc}
\varphi^{\prime}=39^{\circ}, 2 N & H^{\prime}=02^{\mathrm{h}} 45^{\mathrm{m}} 48^{*}, 7 \\
i^{\prime}=15^{\circ}, 1 \mathrm{EGr} . \quad h^{\prime}=0,04 R+33=286,5 \mathrm{~km}(R=6338 \mathrm{~km})
\end{array}
$$

I dati necessari per il calcolo sono riassunti nelle tabelle II e $\mathrm{V}$ e le dromocrome adottate sono quelle di Jeffreys e Bullen grià citate.

Per le radici del sistema normale corrispondente al sistema [12] abluiamo ottenuto i seguenti valori:

$$
\begin{array}{ll}
\delta \varphi^{\prime}=-0^{\circ} 00^{\prime} 10^{\prime \prime} & \delta H^{\prime}=+0^{\prime}, 7 \\
\delta{\nu^{\prime}}^{\prime}=+0^{\circ} 03^{\prime} 41^{\prime \prime} & \delta h^{\prime}=+17,7 \mathrm{~km} \\
\mu_{\rho^{\prime}}= \pm 5^{\prime}, 6 & \mu_{1 \mathrm{I}}{ }^{\prime}= \pm 1,5 \mathrm{sec} \\
\mu_{\lambda^{\prime}}= \pm 5^{\prime}, 5 & \mu_{\mathrm{h}^{\prime}}= \pm 22,7 \mathrm{~km}
\end{array}
$$




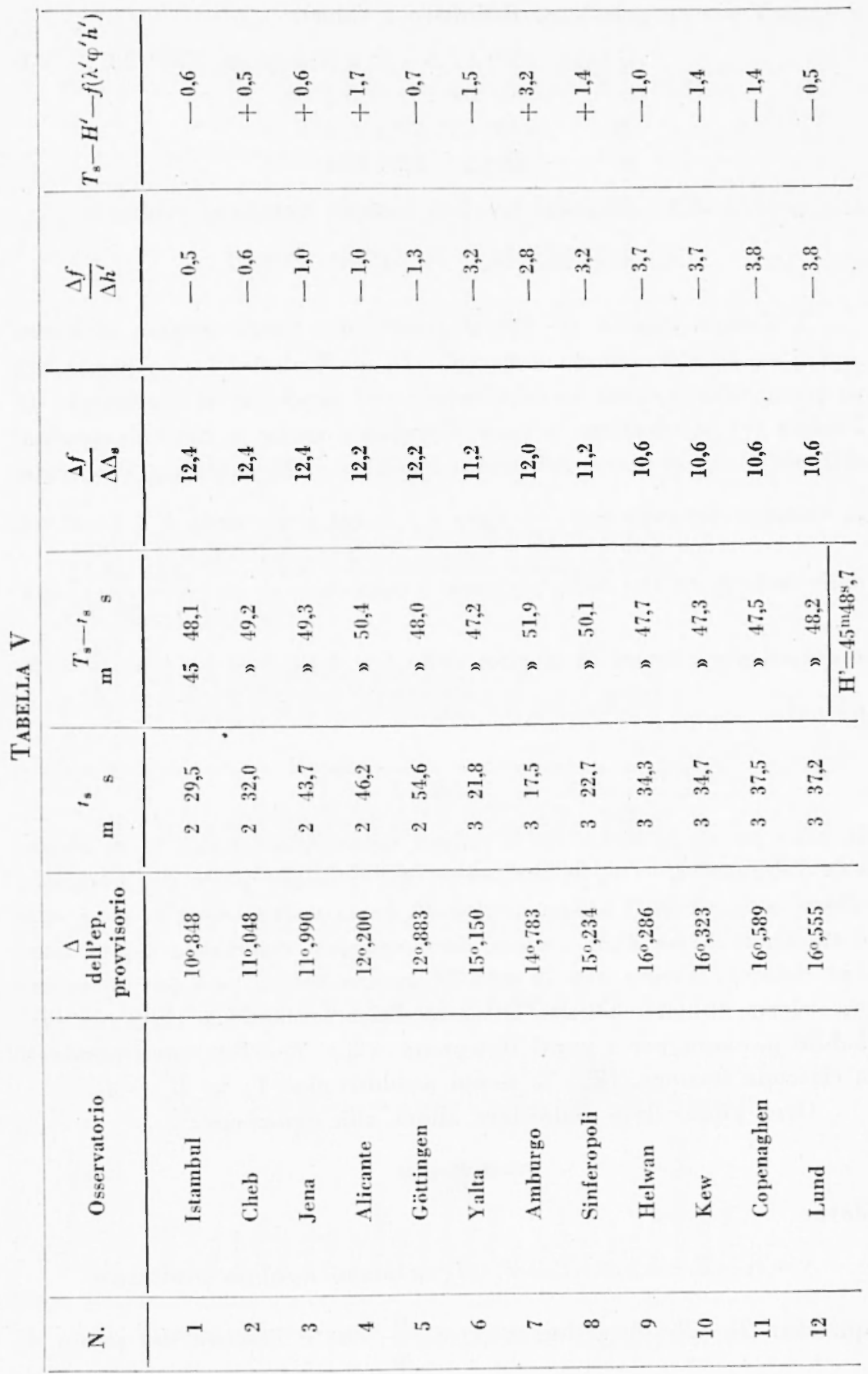


e quindi per le grandezze definitive $\mathrm{i}$ valori:

$$
\begin{array}{ll}
\varphi \text { geoc. } & =39^{\circ} 11^{\prime}, 8+5^{\prime}, 6 \mathrm{~N} \varphi \text { geogr. }=39^{\circ} 23^{\prime}, 2-5^{\prime}, 6 \\
\lambda \quad & =15^{\circ} 09^{\prime}, 7-5^{\prime}, 5 \mathrm{E} \mathrm{Gr} . \\
H \quad & =02^{\mathrm{h}} 45^{\mathrm{m}} 49^{\mathrm{s}}, 4 \mathrm{H}^{\mathrm{s}}, 5 \\
h \quad & =304,2 \pm 22,7 \mathrm{Km}
\end{array}
$$

Il controllo della soluzione ha dato secondo notazioni evidenti :

$$
[v v]=20.3075 \quad\left[l_{1}\right]=20.3077 .
$$

3. Tempo origine. - Per il calcolo del tempo origine ci siamo serviti anche del metodo delle $(S-P)$ di Wadati $\left({ }^{1{ }^{1}}\right)$. La possibilità di poter usare questo metodo risiede nel fatto che il coefficiente di Poisson $(\sigma)$ si mantiene pressoché costante anche a notevole profondità, fin quasi al limite del nucleo terrestre. Dalla costanza di $\sigma$ segue la costanza del rapporto $\frac{V_{\mathrm{P}}}{V_{\mathrm{P}}}$ dove $V_{\mathrm{P}}=$ vel. delle onde $P$ e $V_{\mathrm{S}}=$ vel. delle onde S. Infatti detto rapporto è dato da: $\frac{V_{\mathrm{w}}}{V_{\mathrm{s}}}=\left[\frac{2(1-\sigma)}{1-2_{0}}\right]^{\frac{1}{2}}=K$ e quindi per i tempi di tragitto delle $S$ e delle $P$ si ha pure $\frac{t_{4}}{t_{1}}=K$, da cui :

$$
\frac{t_{1}}{t_{\mathrm{B}}-t_{\mathrm{p}}}=\frac{1}{K-\mathrm{l}}=\mathrm{cost}
$$

In altre parole la linea che si ottiene riportando i tempi $t_{\text {}}$ in ascisse e le differenze $t_{\mathrm{s}}-t_{\mathrm{p}}$ in ordinate è una retta che passa per l'origine; allora, se si vuole il tempo origine $H$, basta vedere dove la retta suddetta taglia l'asse delle ascisse, dove vengono riportati i tempi assoluti delle $P$. Invece che il metodo grafico solito, per una margiore precisione, abbiamo seguito la via analitica cercando la retta più probabile passante per $\mathrm{i}$ punti del piano $\left(T_{1}, T_{\mathrm{s}}-T_{\mathrm{r}}\right)$ corrispondenti a ciascuna stazione. $\left(T_{\mathrm{P}}, T_{\mathrm{s}}\right.$ tempi assoluti $)$ ciò̀ $T_{\mathrm{p}}=H+t_{\mathrm{p}}$.

Ogni punto deve soddisfare allora alla equazione:

$$
y=m x+n
$$

dove:

$$
y=T_{\mathrm{B}^{-}-T_{\mathrm{r}}} \text { e } x=T_{\mathrm{P}}-T_{\mathrm{0}}\left(T_{0}=\text { tempo assoluto arbitrario }\right)
$$

quando: $T_{\mathrm{s}}-T_{\mathrm{P}}=0$ si ha: $x=-\frac{n}{m}$ che è l'ascissa del punto di 


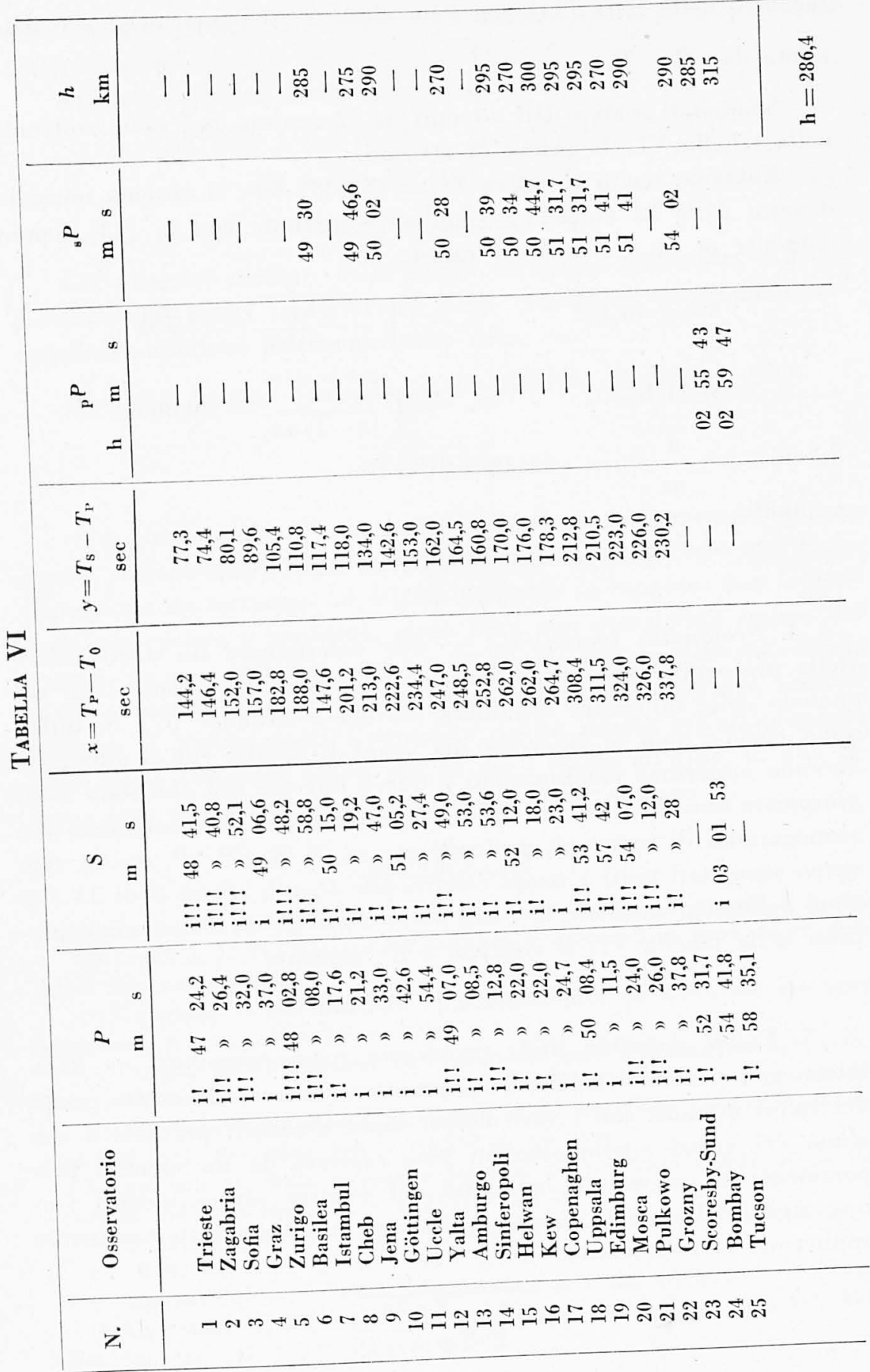


incontro della retta [13] con l'asse della $x$; il tempo origine è dato allora da: $H=T_{0}-\frac{n}{m}$.

Sono stati usati i dati ottenuti da 22 stazioni essi sono contenuti nella tabella VI (le prime 22 stazioni).

Abbiamo scelto il valore $T_{0}=02^{\mathrm{h}} 45^{\mathrm{m}} 00^{*}$; il sistema normale dedotto dalle 22 equazioni simultanee ottenute con la [13] banno lato per $m$ ed $n$ i seguenti valori:

$$
\begin{array}{ll}
n=-43,151 & u_{\mathrm{n}}= \pm \sqrt{\frac{\left[I_{\mathrm{2}}\right]}{(n-1) b b_{1}}}=2,351 \\
m=0,823 & \mu_{\mathrm{m}}= \pm \sqrt{\frac{\left[I_{2}\right]}{(n-1) a a_{1}}}=0,00968
\end{array}
$$

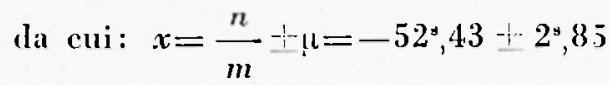

e quindi :

$$
H=T_{0}-\frac{n}{m}-\cdots=02^{\mathrm{h}} 45^{\mathrm{m}} 52^{*}, 4+2^{*}, 8
$$

4. Profondità ipocentrale. - Per determinare un altro valore della profonditá ipocentrale abliamo ritenuto soddisfacente l'uso del metodo delle differenze simultanee dovuto a Brumner (17). Si utilizzano, come è noto, le differenze dei tempi di tragitlo che si otlengono facendo opportune combinazioni a due a due tra fasi che sono maggiomente sensibili alle variazioni di profondita e che compaiono nei sismogrammi di terremoti profondi per es. $P, S, p P$, s $P$, ecc. A tale scopo sono stati usati i tempi relativi alle $P, P P, s P$ ed $S$ di 14 stazioni nelle combinazioni: $S-P, p P-P$. $s P-P$ : i dati sono compresi nella tabella VI; il risultato ì il seguente

$$
h=286,+\therefore 13,4 \mathrm{~km} \text {. }
$$

5. Falori definitivi delle coordinate spazio-temporali. - Assumiamo come valori definitivi delle grandezze ricercate la media pesata dei valori ottenuti con i vari metodi sopra descritt; pertanto a ciascuno dei valori attribuiamo un peso espresso da un numero proporzionale all'inverso del quadrato del relativo errore medio; l'errore medio della media pesata di ciascuna grandeza ì stato otlenuto applicando la relazione:

$$
\mu=\sqrt{\frac{\Sigma p \delta \delta}{\Sigma p(n-1)}}
$$


dove con $p$ viene indicato il peso, $\delta$ lo scarto e con $n$ il numero delle determinazioni esistenti per ogni grandezza.

Abliamo ottenuti i seguenti valori finali:

$$
\begin{gathered}
\text { i. }=15^{\circ} 10^{\prime}, 3=1^{\prime}, 6 E \mathrm{Gr}, \quad \wp=39^{\circ} 21^{\prime}, 6 \div 2^{\prime}, 6 \mathrm{~N} \\
H=02^{\mathrm{h}} 45^{\mathrm{m}} 50^{\mathrm{s}}, 1-1^{\mathrm{s}}, 3 \\
h=290,9-7,8 \mathrm{~km} .
\end{gathered}
$$

Gli ulteriori risultati dello studio di questo terremoto, ottenuti partendo dai valori sopra trovati delle coordinate spazio-temporali, verranno pubblicati in una prossima nota.

Roma - Istituto Nazionale di Geofisica - Novembre 1950.

\section{RIASSUNTO}

E la prima parte di uno studio riguardante un terremoto ad ipocentro profondo verificatosi nel Tirreno Meridionale. Dopo una breve discussione sui terremoti ad origine profonda in rapporto con la geo-logia superficiale e profonda, viene fatta una esposizione critica dev metodi che sono stati qui usati per la determinazione delle coordinate spazio-temporali. Viene confermata la profondità di $300 \mathrm{~km}$, eccezionale per il Moditerraneo. L'epicentro risulta situato sull'allineamento della fascia vulcanica attiva, che va dal Vesuvio allEtna, in una regione sede di una cospicua anomalia isostatica positiva.

\section{BIBLIOGRAFIA}

(1) Strank; Am Erdebebengeographie. Handbuck der Geophysik, vol. 4 Berlin.

(-) Livan J. J., The geographical distribution of deep-focus earthquakes. Bull. Seism. Soc. Am. 36, 1936.

(3) Gutwanbirg B. and Riciten C. F., Seismicity of the Farth and associaled phenomena. Princeton, New Jersey (1949).

(4) Crituos M. A, Relazioni fra i jenomeni sismici e le manifestazioni vulcaniche nel mare Egeo e nella fascia orientale. Geofisica, Milano, vol. 8 pp. 145-159.

(5) Aganexvox: G, Studio macrosismico del terremoto Calabro-Siculo del 13/t/1938. Boll. Soc. Sism. Ital. v. XXXV1 n. 3-t, 1938.

Acanexvoxe G., Sulla profonditi del foco del terremoto Calabro-Siculo del 13/4/1938. Boll. Soc. Sism. Ital. v. XXXVII n. 1.2, 1939.

(ii) Dinutuesce: G., Sur la determination des hypocentres par des observations proches. Boll. Soc. Sism. Ital. v. XXXVII n. 1.2, 1939.

(7) Morthl C., La rete geofisica e geodetica in ltalia. Trieste 1946.

(8) Gutwastig B., Magnitude determination for deep.jocus earthquakes. Bull. Seism. Soc. Am., vol. XXXV, pp. 117-130, 1915. 
(9) Galitzin B, Zur frage der Bestimmung der Herdtieje eines Bebens und der Fortpflanzungsgeschwindigheit des seismischen Wellen in den oberen Erleschichten. C. R. des Sciences de la Commissione Seismique Permanent. 5 (359-130.) 1912.

(10) Gutemberc, B. e Richter C. F., Materials for the study of deep-focus earthqualies. Bull. Seism. Soc. Am. 26 (341-390) 1936.

(11) Brunner G. J, The deep earthquale of May 26, 1932. Gerl. Beitr. z. Geophysik Bd 53 (1938).

(12) Lyich W. A. and Dillon, The deep-focus earthquake of May 19, 1940 in the Sea of Othotsk. Bull. Seism. Soc. Am. v. 33 (251-269) 1943.

(13) Jeffreys H. and Bullen H. E, Seismological Tables. British. Ass. Of Science, London 19.10.

(14) Macelwane, Seismology. New York 1936.

(15) Calor P. e Peronaci F, $1 l$ terremoto del Turkestan del $2 / 11 / 1946$. Amali di Geofisica, v, 1, n. 2, 1948.

(16) Conrie L. J.. The geocentric diretion cosines of Seismological Observatories. British Ass. Adv. Science. London 1938.

(17) Brinnen S. J. and Macklwane J. B., The Brunner focal depth-time-distance. chart. New York 1935.

(18) Wadati K., On the travel time of Earthquthe Mrates. Part II. Geophysical Magazine v. 7, 1933. 